Case Report

\title{
Disseminated Tuberculosis Presenting as Chronic Orchiepididymitis in a Military Trainee: A Case Report and Review of the Literature
}

\author{
Michael U. Williams, ${ }^{1}$ Ashley Burris ${ }^{D},{ }^{2}$ Amy Zingalis, ${ }^{2}$ David A. Lindholm $\left(\mathbb{D},{ }^{3}\right.$ \\ and Brian $\mathrm{K}$. White $\mathbb{I D}^{1,4}$ \\ ${ }^{1}$ Department of Medicine, San Antonio Military Medical Center, Joint Base San Antonio-Fort Sam Houston, \\ TX, USA \\ ${ }^{2}$ Department of Pathology, San Antonio Military Medical Center, Joint Base San Antonio-Fort Sam Houston, \\ TX, USA \\ ${ }^{3}$ Infectious Disease Service, Wright-Patterson Medical Center, Wright-Patterson Air Force Base, OH, USA \\ ${ }^{4}$ Infectious Disease Service, San Antonio Military Medical Center, Joint Base San Antonio-Fort Sam Houston, \\ TX, USA
}

Correspondence should be addressed to David A. Lindholm; david.a.lindholm4.mil@mail.mil

Received 19 May 2018; Accepted 12 July 2018; Published 29 July 2018

Academic Editor: Peter Olumese

Copyright (C) 2018 Michael U. Williams et al. This is an open access article distributed under the Creative Commons Attribution License, which permits unrestricted use, distribution, and reproduction in any medium, provided the original work is properly cited.

\begin{abstract}
Orchiepididymitis is a clinical diagnosis. The acute form secondary to sexually transmitted or enteric pathogens is well known to primary care providers. However, chronic orchiepididymitis may be secondary to genitourinary tuberculosis (TB), and physicians in countries with a low prevalence of TB might not consider it in their differential diagnosis. Indeed, cognitive errors, such as anchoring or availability bias, may contribute to a delayed diagnosis of genitourinary TB. We present a case of chronic orchiepididymitis as a result of disseminated TB in a Cameroonian male who was visiting the United States for military training. He experienced diagnostic delay and was ultimately diagnosed by orchiectomy. Early consideration of a diagnosis of TB for chronic or recurrent orchiepididymitis in a patient with epidemiologic risk factors is of utmost importance because delayed diagnosis could lead to organ loss.
\end{abstract}

\section{Introduction}

Despite advances in diagnostics, treatment, and prevention, tuberculosis (TB) remains a global problem, infecting onethird of the world's population [1]. Mycobacterium tuberculosis is an aerobic bacillus that typically infects humans via inhalation. TB is capable of surviving in a latent state for many years and can spread hematogenously to distant sites [2]. Although genitourinary TB is not an uncommon manifestation of extrapulmonary TB, physicians in countries of low TB prevalence may not consider it in the differential diagnosis of a young male presenting with chronic or recurrent orchiepididymitis. We report an illustrative case of a Cameroonian male who developed chronic orchiepididymitis as a result of disseminated TB, which was diagnosed by orchiectomy while visiting the United States (US) for military training.

\section{Case Presentation}

A 23-year-old male from Cameroon presented with acute-onset, right-sided scrotal pain and swelling while in the US for military training. He denied current or prior sexual activity, associated penile discharge, hematuria, fevers night sweats, weight loss, or cough. Urinalysis revealed pyuria, follow-on culture was not performed, and urine nucleic acid amplification testing (NAAT) was negative for Neisseria gonorrhoeae and Chlamydia trachomatis. He was diagnosed with acute epididymitis and treated with ceftriaxone and doxycycline. His symptoms improved, only 
to recur 6 weeks later, prompting a repeat ultrasound that revealed persistent epididymitis. Repeat urinalysis (no culture) and gonorrhea and chlamydia NAAT were negative. He was given an empiric 30-day course of ciprofloxacin, which improved the pain, but the swelling persisted.

Five months after his initial evaluation, he returned with another recurrence of symptoms. Physical exam again revealed prominent right-sided scrotal swelling and tenderness. An ultrasound demonstrated right-sided epididymitis with possible necrosis, increasing complexity of the associated hydrocele, and new concerns for a focal scrotal abscess. This was further evaluated with a contrasted computed tomography (CT) of the chest, abdomen, and pelvis, which revealed a complex right-sided scrotal fluid collection (Figure 1) in addition to an enlarged and heterogeneous prostate, an enlarged juxtaesophageal lymph node, and right lung apical nodular scarring. Although TB was considered in the differential diagnosis, a unilateral orchiectomy was performed, as malignancy was also high on the differential given his age.

Grossly, the epididymis was enlarged $(6.0 \times 1.7 \times 1.5 \mathrm{~cm}$; Figure 2), with caseating necrosis and miliary deposits within the testicle and epididymis (Figure 3 ). There was an accompanying large scrotal abscess. The histologic sections showed diffuse necrotizing granulomas, with giant cell formation in the scrotum, testis, and epididymis (Figure 4). The testicular parenchyma stained positive for acid-fast bacilli (AFB), and a surgical-scrotal aspirate NAAT using the GeneXpert MTB/RIF assay (Cepheid, Sunnyvale, CA) was positive for Mycobacterium tuberculosis (no rifampin resistance was detected). Urine AFB stain and urine NAAT were negative for TB; ultimately, the urine AFB culture was positive for TB. Despite a normal chest X-ray and reported lack of pulmonary symptoms, his sputum was also positive for TB by stain, NAAT, and culture. Antimicrobial susceptibility testing revealed no resistance to first-line agents. An immunoassay for human immunodeficiency virus (HIV) was nonreactive.

He was started on rifampin, isoniazid, pyrazinamide, and ethambutol. However, due to the development of hepatotoxicity 12 days into therapy without evidence of viral hepatitis, he was transitioned to ethambutol, levofloxacin, and intravenous amikacin. On treatment day 15, his sputum smears converted to negative. After resolution of his druginduced liver injury on treatment day 26, amikacin was discontinued in favor of rifampin, and he was recalled to Cameroon soon thereafter on an all-oral, three-drug regimen.

\section{Discussion}

Extrapulmonary TB accounted for $18.7 \%$ of TB cases in the US from 1993 to 2006; of those extrapulmonary cases, 6.5\% involved the genitourinary tract [3], although genitourinary involvement has been reported to be present in as high as $42.9 \%$ of cases in other countries [4]. The diagnosis of genitourinary $\mathrm{TB}$ is challenging and requires a high level of clinical suspicion based on the epidemiologic context. Given the low prevalence of TB in the US and the fact that patients with extrapulmonary TB often lack the classic symptoms of pulmonary TB, US physicians may have a low clinical suspicion for genitourinary TB [5]. Unfortunately, the late diagnosis of $\mathrm{TB}$ orchiepididymitis can result in organ loss [6].

Risk factors for genitourinary TB include foreign birth, HIV infection, hemodialysis, and kidney transplantation [7-9]. In 2016, over 9,000 TB cases were reported in the US, and $68 \%$ of the cases occurred among foreign-born persons, making this a key trigger to raise one's clinical suspicion [10]. Additionally, suspicion for TB should be higher in patients whose symptoms fail to resolve or who have recurrent symptoms after empiric treatment for common causes of acute epididymitis [8].

The diagnostic delay in our patient was primarily the result of failing to consider TB in the differential diagnosis. In clinical practice, cognitive error is more common than knowledge gap error and commonly leads to misdiagnosis [11]. Cognitive errors (e.g., anchoring, availability bias, representativeness bias, and premature closure) likely contributed to his delayed diagnosis. Chronic orchiepididymitis is variably defined as at least 6 weeks to 3 months of discomfort and/or pain localized to the scrotum $[12,13]$. In a patient presenting with such a clinical syndrome, it is important to maintain a broad differential diagnosis, including both infectious and noninfectious causes: TB, bacillus Calmette-Guérin, filariasis, leishmaniasis, brucellosis, syphilis, chlamydia, paracoccidioidomycosis, malignancy, autoimmune diseases, obstruction, medication adverse effects, trauma, postinfectious, and idiopathic [12-15]. Noninvasive tests directed by epidemiologic clues, such as three or more early-morning urine AFB cultures [8] in a foreign-born male, may allow for early diagnosis and successful pharmacotherapy, eliminating the need for orchiectomy. However, testicular malignancies can present similarly, are most common among males 15 to 34 years of age [16], and may be cured by surgical excision [17-19]. If a noninvasive approach fails to diagnose genitourinary TB, surgery may be necessary in order to confirm the diagnosis and rule out malignancy [6].

Although the GeneXpert assay is approved by the Food and Drug Administration for pulmonary specimens only, its use as a tool in the diagnosis of extrapulmonary TB has been studied. Meta-analyses show a wide range of sensitivity (0$100 \%$, median $22-83 \%$ ) and a narrower range of specificity (73-100\%, median 98-100\%) [20-22]. The variability in sensitivity is secondary to differences in study design, patient population, gold standard, sample processing, anatomic source of the sample, tissue or fluid type of the sample, sample volume, nonhomogeneous distribution of mycobacteria within the sample, mycobacterial burden, and AFB smear status [9,20-22]. Although it should not be used to rule out extrapulmonary TB given its poor sensitivity, the off-label use of the Xpert assay can be very helpful in the rapid detection of extrapulmonary TB given its high specificity and its applicability to samples obtained via noninvasive (e.g., voided) or minimally invasive techniques (e.g., image-guided aspiration) $[21,22]$. Early diagnosis can facilitate early therapy and-in some cases-may even prevent premature surgery. In our 


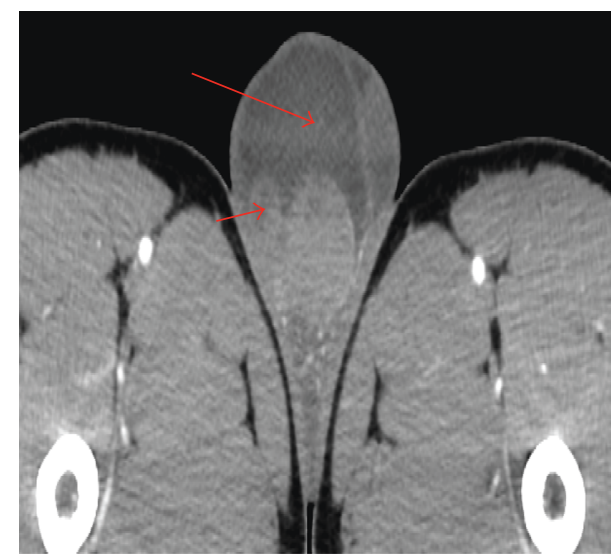

FIgURE 1: Axial computed tomography (CT) image, depicting a heterogeneous right testicle, with associated right scrotal fluid collection (long arrow) and inflamed right epididymis (short arrow).

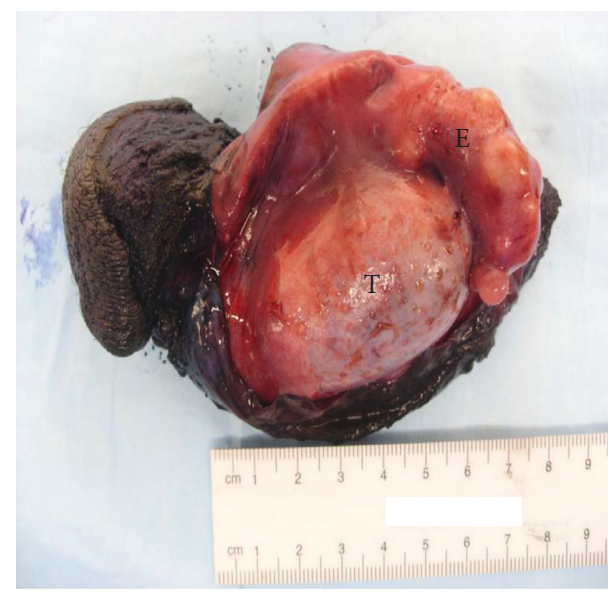

Figure 2: Opened scrotal sac with the exposed testicle (T) and enlarged and nodular epididymis (E).

patient, the urine NAAT was negative, but the NAAT from the surgical scrotal aspirate was positive and triggered the initiation of anti-TB therapy.

Our case highlights the importance of maintaining a broad differential diagnosis to avoid cognitive errors and using the patient's epidemiologic context to increase clinical suspicion for genitourinary TB. Although not pursued in our case early enough to avoid surgery, early consideration of microbiologic or molecular testing of nonsurgical specimens may facilitate diagnosis and optimize outcomes.

\section{Disclosure}

The content of this publication is the sole responsibility of the authors and does not necessarily reflect the views or policies of the Wright-Patterson Medical Center, the Brooke Army Medical Center, the U.S. Army Medical Department, the U.S. Army Office of the Surgeon General, the Department of the Army, the Department of the Air Force, the Department of Defense, or the U.S. Government. Mention of

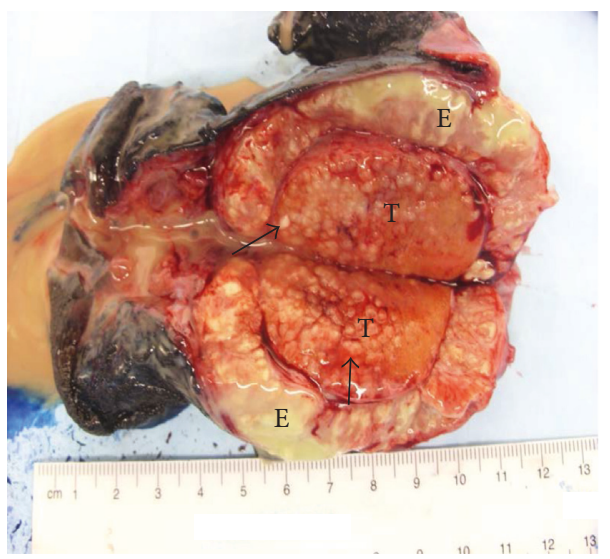

FIgUre 3: Bisected epididymis (E) and testicle (T), depicting a yellow-tan exudate and miliary deposits (arrows).

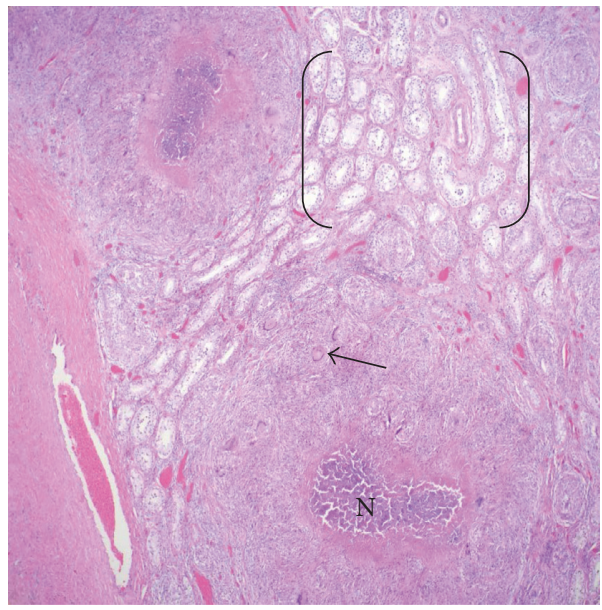

FIGURE 4: H\&E, 4x. Necrotizing granulomatous inflammation in a miliary-type distribution, with multinucleated giant cells (histiocytes, arrow), central necrosis $(\mathrm{N})$, and intervening, uninvolved, normal testicular tubules (brackets).

trade name, commercial products, or organizations does not imply endorsement by the U.S. Government. This case report was presented in part at the Texas Chapter of the American College of Physicians (Houston, TX, November 2016) and the Texas Society of Pathologists 96th Annual Meeting (Bastrop, TX, January 2017).

\section{Conflicts of Interest}

The authors are employees of the U.S. Government. This work was prepared as part of their official duties. Title $17 \mathrm{U}$. S.C. 105 provides that "copyright protection under this title is not available for any work of the United States Government." Title 17 U.S.C. 101 defines a U.S. Government work as a work prepared by a military service member or an employee of the U.S. Government as part of that person's official duties. The authors declare that they have no conflicts of interest. 


\section{References}

[1] A. Abbara and R. N. Davidson, "Etiology and management of genitourinary tuberculosis," Nature Reviews Urology, vol. 8, no. 12, pp. 678-688, 2011.

[2] A. O. Ankrah, T. S. van der Werf, E. F. de Vries, R. A. Dierckx, M. M. Sathekge, and A. W. Glaudemans, "PET/CT imaging of Mycobacterium tuberculosis infection," Clinical and Translational Imaging, vol. 4, no. 2, pp. 131-144, 2016.

[3] H. M. Peto, R. H. Pratt, T. A. Harrington, P. A. LoBue, and L. R. Armstrong, "Epidemiology of extrapulmonary tuberculosis in the United States, 1993-2006," Clinical Infectious Diseases, vol. 49, no. 9, pp. 1350-1357, 2009.

[4] E. Kulchavenya, "Extrapulmonary tuberculosis: are statistical reports accurate?," Therapeutic Advances in Infectious Disease, vol. 2, no. 2, pp. 61-70, 2014.

[5] J. N. Sama, N. Chida, R. M. Polan, J. Nuzzo, K. Page, and M. Shah, "High proportion of extrapulmonary tuberculosis in a low prevalence setting: a retrospective cohort study," Public Health, vol. 138, pp. 101-107, 2016.

[6] E. Kulchavenya and D. Kholtobin, "Diseases masking and delaying the diagnosis of urogenital tuberculosis," Therapeutic Advances in Urology, vol. 7, no. 6, pp. 331-338, 2015.

[7] L. Norbis, R. Alagna, E. Tortoli, L. R. Codecasa, G. B. Migliori, and D. M. Cirillo, "Challenges and perspectives in the diagnosis of extrapulmonary tuberculosis," Expert Review of Anti-Infective Therapy, vol. 12, no. 5, pp. 633-647, 2014.

[8] J. Yuan, "Genitourinary presentation of tuberculosis," Reviews in Urology, vol. 17, no. 2, pp. 102-105, 2015.

[9] P. K. Mehta, A. Raj, N. Singh, and G. K. Khuller, "Diagnosis of extrapulmonary tuberculosis by PCR," FEMS Immunology and Medical Microbiology, vol. 66, no. 1, pp. 20-36, 2012.

[10] K. M. Schmit, Z. Wansaula, R. Pratt, S. F. Price, and A. J. Langer, "Tuberculosis-United States, 2016," Morbidity and Mortality Weekly Report, vol. 66, no. 11, pp. 289-294, 2017.

[11] G. R. Norman and K. W. Eva, "Diagnostic error and clinical reasoning," Medical Education, vol. 44, no. 1, pp. 94-100, 2010.

[12] J. C. Nickel, "Chronic epididymitis: a practical approach to understanding and managing a difficult urologic enigma," Reviews in Urology, vol. 5, no. 4, pp. 209-215, 2003.

[13] M. Çek, L. Sturdza, and A. Pilatz, "Acute and chronic epididymitis," European Urology Supplements, vol. 16, no. 4, pp. 124-131, 2017.

[14] O. de Cassio Saito, N. de Barros, M. C. Chammas, I. R. S. Oliveira, and G. G. Cerri, "Ultrasound of tropical and infectious diseases that affect the scrotum," Ultrasound Quarterly, vol. 20, no. 1, pp. 12-18, 2004.

[15] K. A. Workowski, "Centers for disease control and prevention sexually transmitted diseases treatment guidelines," Clinical Infectious Diseases, vol. 61, no. 8, pp. S759-S762, 2015.

[16] P. Crawford and J. A. Crop, "Evaluation of scrotal masses," American Family Physician, vol. 89, no. 9, pp. 723-727, 2014.

[17] M. Chovanec, N. Hanna, K. C. Cary, L. Einhorn, and C. Albany, "Management of stage I testicular germ cell tumours," Nature Reviews Urology, vol. 13, no. 11, pp. 663-673, 2016.

[18] M. J. Murray, R. A. Huddart, and N. Coleman, "The present and future of serum diagnostic tests for testicular germ cell tumours," Nature Reviews Urology, vol. 13, no. 12, pp. 715$725,2016$.

[19] A. L. Kühn, E. Scortegagna, K. M. Nowitzki, and Y. H. Kim, "Ultrasonography of the scrotum in adults," Ultrasonography, vol. 35, no. 3, pp. 180-197, 2016.
[20] S. D. Lawn and A. I. Zumla, "Diagnosis of extrapulmonary tuberculosis using the Xpert ${ }^{\circledR}$ MTB/RIF assay," Expert Review of Anti-Infective Therapy, vol. 10, no. 6, pp. 631-635, 2012.

[21] L. Maynard-Smith, N. Larke, J. A. Peters, and S. D. Lawn, "Diagnostic accuracy of the Xpert MTB/RIF assay for extrapulmonary and pulmonary tuberculosis when testing non-respiratory samples: a systematic review," BMC Infectious Diseases, vol. 14, no. 1, p. 709, 2014.

[22] C. M. Denkinger, S. G. Schumacher, C. C. Boehme, N. Dendukuri, M. Pai, and K. R. Steingart, "Xpert MTB/RIF assay for the diagnosis of extrapulmonary tuberculosis: a systematic review and meta-analysis," European Respiratory Journal, vol. 44, no. 2, pp. 435-446, 2014. 


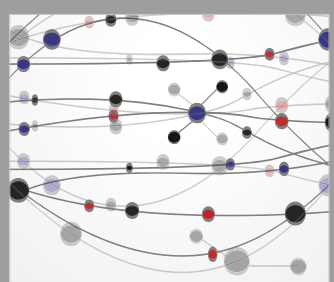

The Scientific World Journal
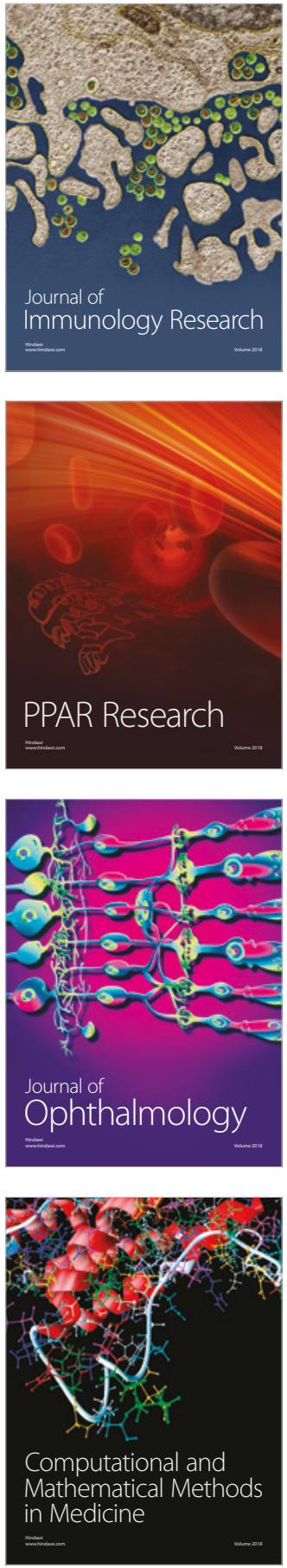

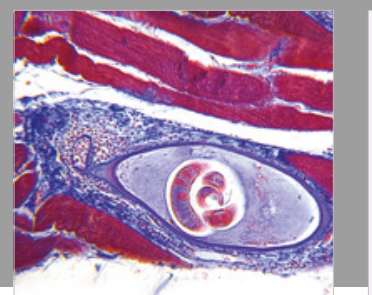

Gastroenterology Research and Practice

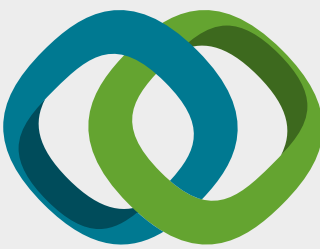

\section{Hindawi}

Submit your manuscripts at

www.hindawi.com
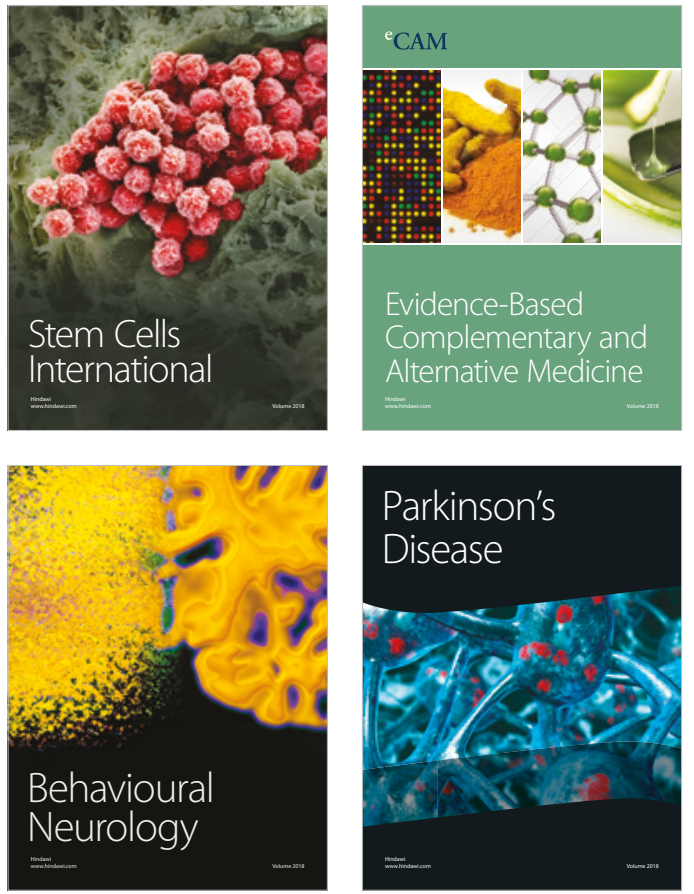

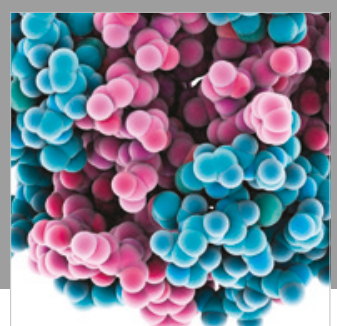

ournal of

Diabetes Research

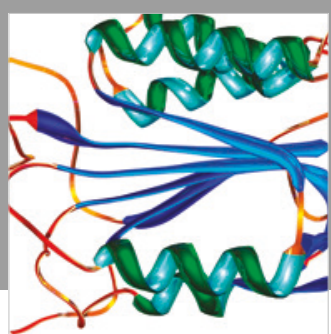

Disease Markers
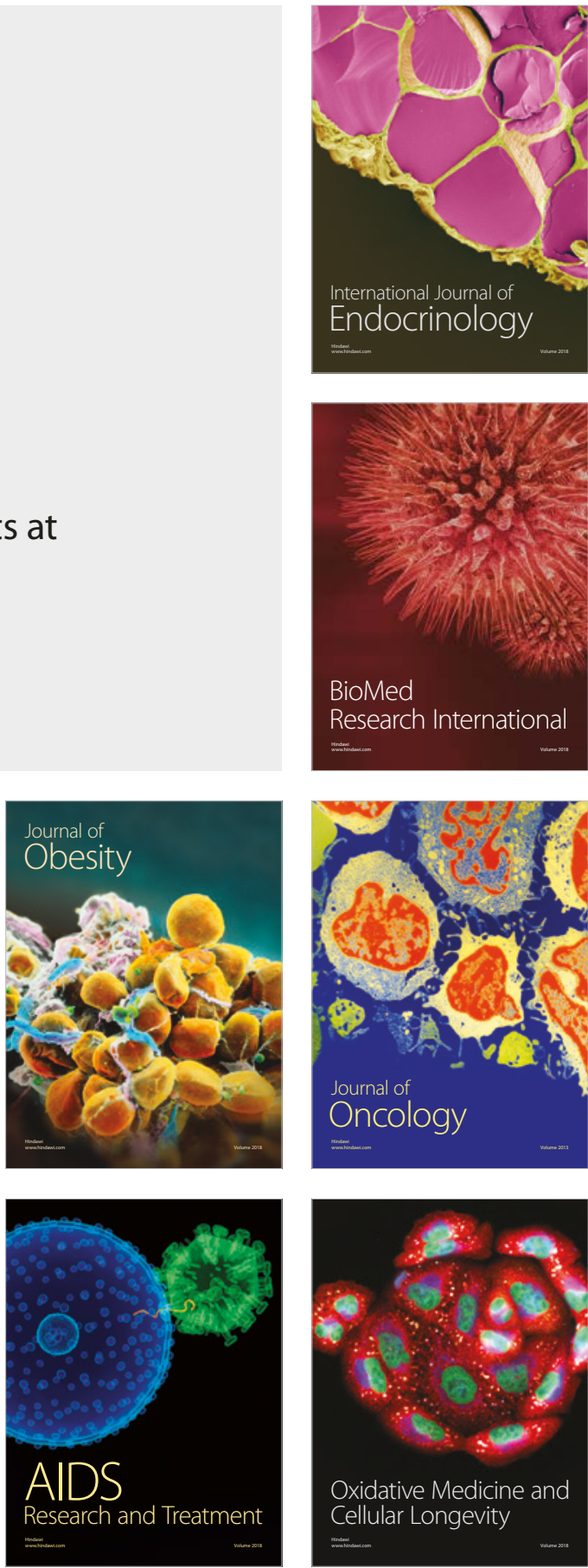